\title{
Molecular Genetic Analysis of Sexual Rejection: Roles of Octopamine and Its Receptor OAMB in Drosophila Courtship Conditioning
}

\author{
Chuan Zhou, ${ }^{1,2 *}$ Hai Huang, ${ }^{1 \star}$ Susy M. Kim, ${ }^{3 *}$ Hsuanwen Lin, ${ }^{4}$ Xianglan Meng, ${ }^{6}$ Kyung-An Han, ${ }^{5}$ Ann-Shyn Chiang, ${ }^{4}$ \\ Jing W. Wang, ${ }^{3}$ Renjie Jiao, ${ }^{1}$ and Yi Rao ${ }^{2,6}$ \\ ${ }^{1}$ Institute of Biophysics and the Graduate School of the Chinese Academy of Sciences, Beijing 100101, People's Republic of China, ${ }^{2}$ National Institute of \\ Biological Sciences, Beijing 102206, People's Republic of China, ${ }^{3}$ Neurobiology Section, Division of Biological Sciences, University of California, San Diego, \\ La Jolla, California 92093, ${ }^{4}$ Brain Research Center \& Institute of Biotechnology, National Tsinghua University, Hsinchu, Taiwan 30013, Republic of China, \\ ${ }^{5}$ Department of Biological Sciences, Border Biomedical Research Center, University of Texas at El Paso, El Paso, Texas 79968, and ${ }^{6}$ Peking-Tsinghua Center \\ for Life Sciences at Peking University School of Life Sciences, Beijing 100871, People's Republic of China
}

After Drosophila males are rejected by mated females, their subsequent courtship is inhibited even when encountering virgin females. Molecular mechanisms underlying courtship conditioning in the CNS are unclear. In this study, we find that tyramine $\beta$ hydroxylase $(\mathrm{T} \beta \mathrm{H})$ mutant males unable to synthesize octopamine $(\mathrm{OA})$ showed impaired courtship conditioning, which could be rescued by transgenic $\mathrm{T} \beta \mathrm{H}$ expression in the CNS. Inactivation of octopaminergic neurons mimicked the $\mathrm{T} \beta \mathrm{H}$ mutant phenotype. Transient activation of octopaminergic neurons in males not only decreased their courtship of virgin females, but also produced courtship conditioning. Single cell analysis revealed projection of octopaminergic neurons to the mushroom bodies. Deletion of the OAMB gene encoding an OA receptor expressed in the mushroom bodies disrupted courtship conditioning. Inactivation of neurons expressing OAMB also eliminated courtship conditioning. OAMB neurons responded robustly to male-specific pheromone cis-vaccenyl acetate in a dose-dependent manner. Our results indicate that $\mathrm{OA}$ plays an important role in courtship conditioning through its OAMB receptor expressed in a specific neuronal subset of the mushroom bodies.

\section{Introduction}

Although Drosophila males are born with the capacity of displaying stereotypically sequential courtship rituals, male courtship behavior is also subject to modification by social experience. After prior experience of pairing with unreceptive mated females who may run away from, or kick, males, courtship behavior of male flies toward females will be suppressed, so that they would later be less interested in females, even when presented with virgin females, normally highly attractive to adult males (Siegel and Hall, 1979).

Courtship conditioning involves multiple chemosensory cues, including volatile appetitive and aversive pheromonal cues (Mehren et al., 2004; Ejima et al., 2005, 2007; Siwicki et al., 2005).

Received Feb. 2, 2012; revised July 23, 2012; accepted July 26, 2012.

Author contributions: C.Z., A.-S.C., J.W.W., R.J., and Y.R. designed research; C.Z., H.H., S.M.K., H.L., and X.M. performed research; K.-A.H. contributed unpublished reagents/analytic tools; C.Z., A.-S.C., J.W.W., R.J., and Y.R. analyzed data; C.Z. and Y.R. wrote the paper.

This work was supported by a grant from the Ministry of Science and Technology (973 program No. 2010(B833900).

We are grateful to Drs. Paul Garrity, Kristin Scott, Tzumin Lee, Jay Hirsh, Maria Monastirioti, Ping Shen, and Chun-Fang Wu and the Bloomington Stock Center for their flies; and to members of the Rao Lab for discussion.

${ }^{*}$ C.Z., H.H., and S.M.K. contributed equally to this work.

Correspondence should be addressed to Yi Rao, Peking-Tsinghua Center for Life Sciences at Peking University School of Life Sciences, 5 Yiheyuan Road, Beijing 100871, People's Republic of China. E-mail:yrao@pku.edu.cn. DOI:10.1523/JNEUROSCI.0517-12.2012

Copyright $\odot 2012$ the authors $\quad 0270-6474 / 12 / 3214281-07 \$ 15.00 / 0$
By comparing the pheromone profile of virgins and mated females, a single chemical component cis-vaccenyl acetate (cVA), has been shown to effectively repress courtship behavior of males by mated females (Ejima et al., 2007). 9-Pentacosene (9-P) may serve as an aphrodisiac cue in the conditioned response of males toward mated females (Siwicki et al., 2005). It is thus conceivable that aphrodisiac and anti-aphrodisiac chemosensory cues are critically required for the formation of courtship conditioning memory.

Genes involved in olfaction learning also play a role in courtship conditioning, suggesting that courtship conditioning shares a common biochemical pathway, at least in part, with olfaction conditioning (Siegel and Hall, 1979; Gailey et al., 1984; Griffith et al., 1993; Joiner and Griffith, 1997). It is not surprising to find that the mushroom bodies, which are known to be important for olfactory learning and memory, are involved in courtship conditioning (Joiner and Griffith, 1999; McBride et al., 1999; Sitnik et al., 2003). It is unclear, however, how sensory inputs are relayed to mushroom bodies during courtship learning.

Octopamine (OA) is an insect equivalent of the mammalian noradrenaline. There are four known OA receptors in Drosophila, one of which is $\mathrm{OAMB}$, an OA receptor in the mushroom bodies. It was found that $\mathrm{OA}$ is involved in appetitive olfactory learning by mediating reward unconditioned stimuli (Schwaerzel et al., 2003). Involvement of the OA pathway in aversive learning is unknown. Recent studies also suggested that OA is important for social behaviors such as aggression and courtship (Certel et al., 
2007, 2010; Zhou et al., 2008). Here we provide evidence that OA plays an important role in courtship conditioning by mediating the aversive input to downstream mushroom body neurons expressing OAMB.

\section{Materials and Methods}

Fly culture. Flies were normally reared at $25^{\circ} \mathrm{C}$ and $60 \%$ humidity and entrained in a $12 \mathrm{~h}$ light/dark condition except in shibire ${ }^{t s}$ or TrpA experiments. L5076-LexA and L5251-LexA are enhancer trap lines made in the Chiang Lab. $\mathrm{T} \beta \mathrm{h}^{\mathrm{nM} 18}$ deletion mutants were a gift from C.-F. Wu (University of Iowa, IA). UAS-T $\beta \mathrm{H}$ was generously provided by M. Monastirioti (Institute of Molecular Biology and Biotechnology, Greece). Tdc2-Gal4 was a gift from J. Hirsh (University of Virginia, VA). UAS-shi ${ }^{\text {ts }}$ was a gift from P. Shen (University of Georgia). UAS-TrpA was a gift from P. Garrity (Brandeis University, MA). UAS-mCD8GFP, 20XUASGCaMP3, and c739-Gal4 were obtained from Bloomington Stock Center. UAS-CD4:: spGFP1-10,LexAop-CD4::spGFP11 was a gift from K. Scott (University of California, Berkeley, CA). LexAop-rCD2::GFP was a gift from T. Lee (Janelia Farm, Ashburn, VA).

oamb knock-in flies and UAS-oamb rescue flies. The genomic segment of oamb gene was cloned into the pTARG vector. The upstream fraction around oamb ATG start codon was amplified by $5^{\prime}$-AAACTACGCGTCCAGCTA ATTGGCGCCAAC-3' and 5'-TTCAATTCGC GGCCGCACTTTTGAGATGGGTGTG-3', while the downstream fraction was amplified by $5^{\prime}$-TC AAAAGTGCGGCCGCGAATTGAAACAGAG TGCGAG-3' and $5^{\prime}$-GTAAAGATCTCTCTTT AGCCGCCTCCAAATGTGTG-3'. The CCGC CATG sequence (oamb start codon is marked) was replaced by a NotI enzyme restriction site, where a Gal4 coding sequence was inserted subsequently. Oligonucleotides used to generate an I-SceI recognition site were AATTTAGGGATA ACAGGGTAAT and

AATTATTACCCTGTTATCCCTA.

The knock-in flies were generated by "ends-in" targeting strategy (Rong and Golic, 2000). w-; OAMB-Gal4 strain was outcrossed into Canton $\mathrm{S}$ background and made red-eyed before behavioral test.

The cDNA of oamb in pcDNA1 vector was cloned and introduced into pUAST vector by EcoRI/XbaI double digestion (Han et al., 1998). The embryo transformation was performed following the standard procedure.

Sample preparation for immunostaining and GFP reconstitution across synaptic partners. Samples were dissected in PBS and fixed in 4\% paraformaldehyde in PBS before being placed in PBS containing 1\% Triton $\mathrm{X}-100$ and $10 \%$ normal goat serum (PBS-T) and degassed in a vacuum chamber to expel tracheal air with six cycles. Next, the brain samples were incubated in PBS-T containing either 1:50 mouse 4F3 anti-discs large antibody (DSHB) or 1:50 mouse nc82 antibody (DSHB) and 1:500 rabbit anti-green fluorescent protein (GFP) antibody (Invitrogen) at $4^{\circ} \mathrm{C}$ for 2 d. After washing with PBS-T three times, samples were incubated in PBS-T containing 1:200 biotinylated goat anti-mouse IgG biotin (Invitrogen) and goat anti-rabbit IgG biotin (Invitrogen) at $4^{\circ} \mathrm{C}$ overnight. Samples were then washed and incubated with 1:500 Alexa Fluor 635 streptavidin (Invitrogen) at $4^{\circ} \mathrm{C}$ overnight. Finally, after extensive washing, the immunolabeled brain samples were directly cleared in FocusClear (CelExplorer) for 5 min and then mounted in a drop of MountClear (CelExplorer) between two Whitney test.
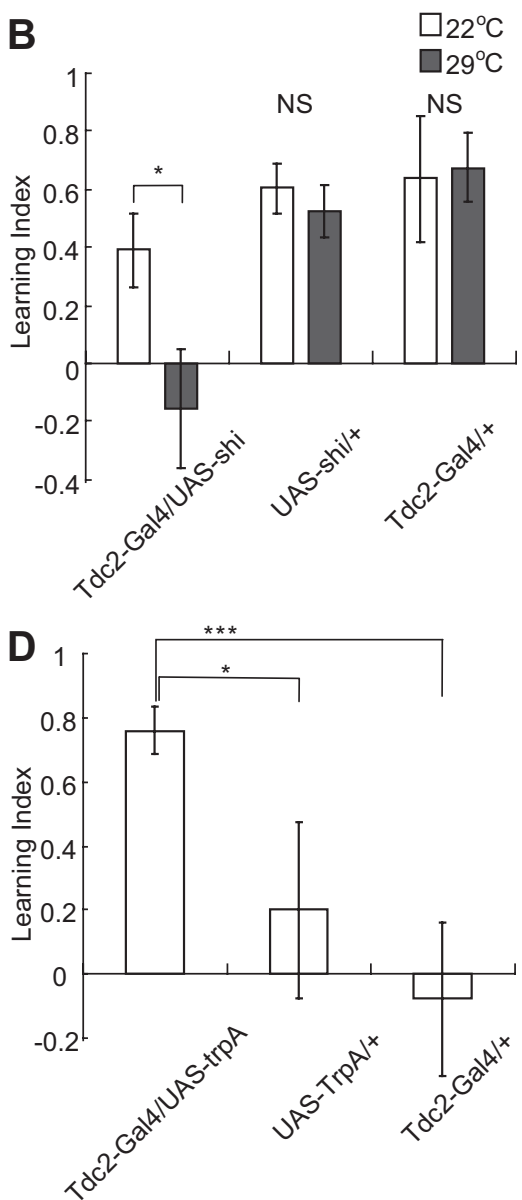

Figure 1. $\quad 0 A$ and octopaminergic neurons are involved in courtship conditioning. $A$, LI of indicated genotypes after courtship conditioning; wt denotes wild-type while $\mathrm{m}$ denotes $\mathrm{T} \beta \mathrm{H}$ mutant background. $N=18,18,18,29$, and 36 for each genotype, reduced courtship conditioning. $N=37,34,33,19,21$, and 21 respectively; ${ }^{*} p<0.05$, NS $p>0.05$, Mann-Whitney test. $\boldsymbol{C}$ Activation of octopaminergic neurons suppresses male-female courtship. $N=24,22,24,18,24$, and 24 , respectively; ${ }^{* * *} p<$ 0.05, Mann-Whitney test. D, Activation of octopaminergic neurons when male flies were paired with virgin females produced a memory trace of courtship repression. $N=38,29$, and 26 for each genotype; ${ }^{*} p<0.05$, ${ }^{* * *} p<0.001$, Mann-

coverslips separated by a spacer ring of $\sim 200 \mu \mathrm{m}$ thickness, so the brain sample was not flattened.

MARCM clones. The genotype for generating MARCM clones of octopaminergic neurons was as follows: hs-FLP,FRT19A,tubP-GAL80/ FRT19A,UAS-mCD8::GFP;Tdc2-GAL4/+;+.

Embryos were given heatshock for $40 \mathrm{~min}$ in $37^{\circ} \mathrm{C}$ water bath to generate Tdc2-F-000007 clones (see Fig. 5B). Tdc2-F-200034 clones were obtained by heat shocking the larvae at $24-48 \mathrm{~h}$ (day 2 ) at $37^{\circ} \mathrm{C}$ for 45 min (Fig. 5C).

Two-photon calcium imaging. Calcium imaging was performed with a custom-built two-photon microscope as described previously (Wang et al., 2003). Live fly preparations were made by taping over the dorsal aspect of the fly head, mounting the fly to a small dish, filling the dish with calcium-free saline, and then cutting away a window through the cuticle lying over the dorsal brain. Calcium-free saline was subsequently replaced with saline containing $2 \mathrm{~mm}$ calcium.

cVA (Cayman Chemicals) was delivered through a custom-made device that consisted of two cartridges. One cartridge was empty and the other held $1 \mu \mathrm{l}$ of cVA placed on a piece of filter paper. Air passing through the two cartridges converged through a fork and was delivered through a single piece of tubing located $5-10 \mathrm{~mm}$ from the antennae. A constant airflow of $1 \mathrm{~L} / \mathrm{min}$ was applied to the antennae via a pipe of 12 $\mathrm{mm}$ diameter and the empty cartridge on the custom device. Odor onset was controlled by mixing a defined percentage of carrier air with air 
A
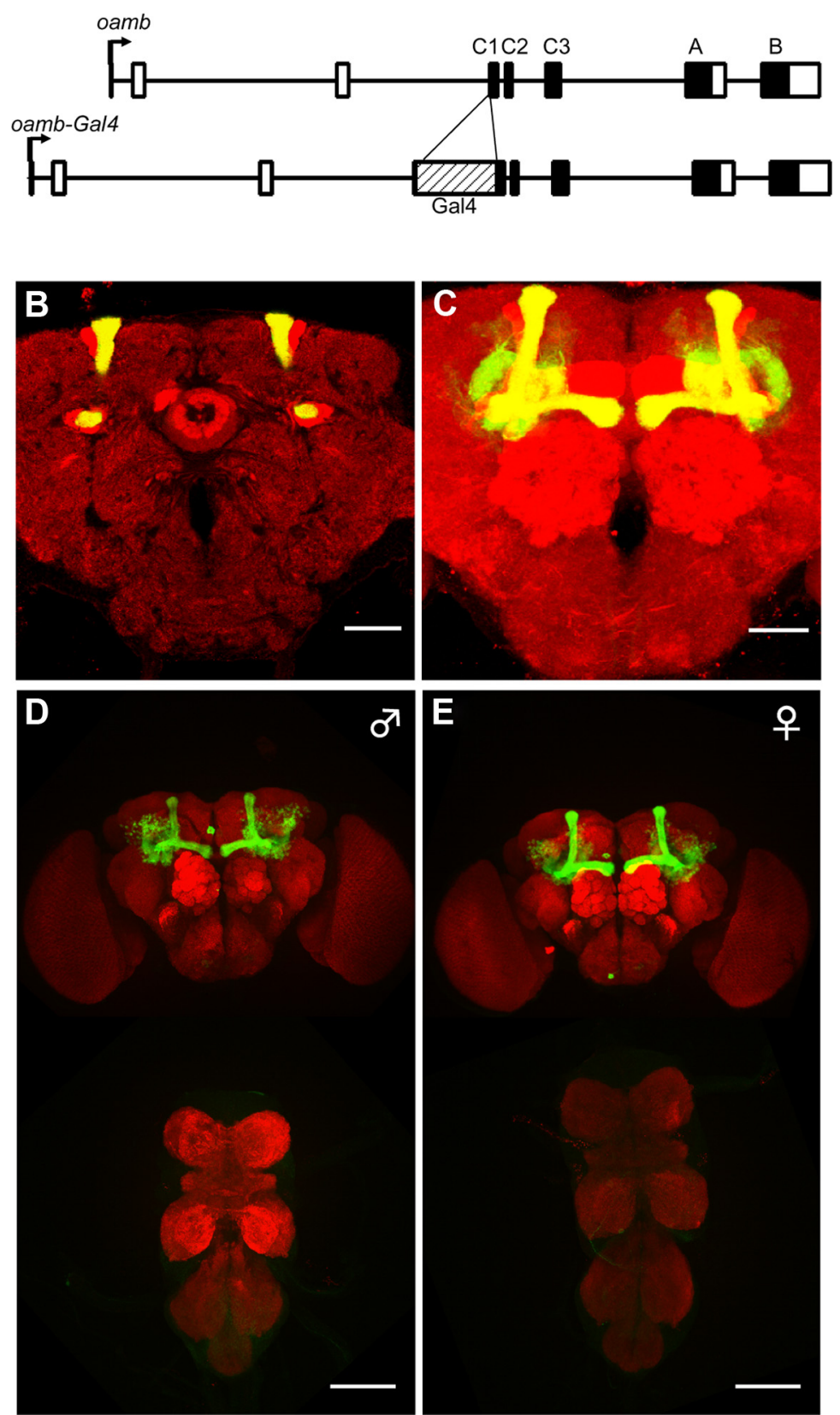

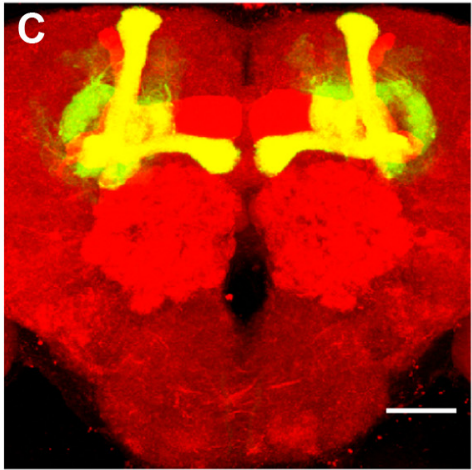

$\mathrm{E}$

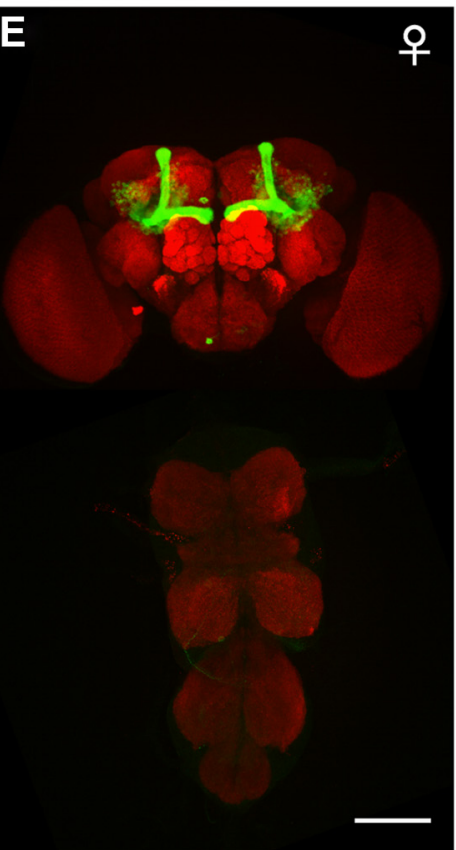

Figure 2. OAMB-Gal4 drives expression in mushroom body $\alpha / \beta$ lobes. The OAMB-Gal4 showed specific expression in $\alpha / \beta$ lobes of the mushroom bodies but not in $\alpha^{\prime} / \beta^{\prime}$ or $\gamma$ lobes. $A$, Schematic of OAMB-Gal4 knock-in in oamb locus. Arrows indicate transcriptional start sites. Black boxes indicate coding sequences. C1-C3 indicate the common exons. A and B are alternative 3' exons. $\boldsymbol{B}$, An anterior confocal section of a male OAMB-Gal4/UAS-mCD8-GFP adult fly counterstained with the anti-disc large antibody (red). Scale bar, $50 \mu \mathrm{m}$. C, Three-dimensional reconstruction image of a male 0AMB-Gal4/UAS-mCD8-GFP adult fly brain Scale bar, $50 \mu \mathrm{m}$. D, Three-dimensional reconstruction image of brain and ventral nerve cord (VNC) of oamb-Gal4/UAS-mCD8GFP (green) adult male counterstained by nc 82 antibody (red). Scale bar, $100 \mu \mathrm{m}$. $\boldsymbol{E}$, Three-dimensional reconstruction image of brain and VNC of oamb-Gal4/UAS-mCD8GFP (green) adult female counterstained by nc82 antibody (red). Scale bar, $100 \mu \mathrm{m}$.

redirected through the odor cartridge on the custom device. Flies received two odor presentations of cVA in order of descending concentration. Upon completion of this schedule, flies received an additional presentation at the highest odor concentration. If this final response was $<10 \%$ of the original value, data from that fly was not used. $\Delta F / F$ values in regions of interest were calculated using an IgorPro custom macro as previously described (Wang et al., 2003). Specifically, after subtraction of average background noise values from all image frames, mean Fo values were obtained from frames of the region of interest before odor stimulation. $\Delta F$ values were calculated from $F_{(t)}-$ mean $F o$ and then normalized to the prestimulation baseline fluorescence. For somatic cell responses, only those cells exhibiting $\Delta F / F$ response amplitudes that were at least two SDs above the average baseline in two odor presentations were deemed responsive to the odorant.

Courtship conditioning assay. All the behavioral assays were performed under $25^{\circ} \mathrm{C}$ and $50-60 \%$ humidity unless indicated otherwise. Male and virgin flies were isolated immediately after eclosion and aged for 5-7 d. A single male was then paired with a virgin in an Eppendorf tube to allow copulation. Those pairs successfully copulated within $2 \mathrm{~h}$, were marked, and the mated females were selected and further aged for 18-24 h before courtship repression training. Male flies were isolated for 5-7 d before paired with a mated female in a chamber to allow $1 \mathrm{~h}$ training. The trained fly or naive fly without pairing with a mated female was then transferred into a new chamber containing a decapitated virgin for test. The first $10 \mathrm{~min}$ of the testing period was videotaped and analyzed for courtship activity the males displayed to the decapitated tester. Courtship index (CI) was the fraction of summed duration of courtship activity during the total $10 \mathrm{~min}$ videotaping period. Learning index was then calculated as $\mathrm{LI}=\left(\mathrm{CI}_{\text {naive }}-\mathrm{CI}_{\text {trained }}\right) / \mathrm{CI}_{\text {naive }} \cdot \mathrm{CI}_{\text {trained }}$ means the CI of trained flies. To calculate $\mathrm{CI}_{\text {naive }}, 12-24$ naive males were tested against decapitated virgins for each genotype. Thus the $\mathrm{CI}_{\text {naive }}$ and $\mathrm{CI}_{\text {trained }}$ were calculated from two populations of flies of the same genotype. A mean LI was then calculated based on individual LI scores.

Statistical analyses were performed with Prism 4 software (GraphPad). Mann-Whitney test or one-way ANOVA was performed to analyze the data. A post hoc Dunnett test was followed to identify significantly different groups. $N$ indicates the number of trained flies for each genotype. Error bars indicate SEM.

Shibire and TrpA experiments. Tdc2-Gal4/ UAS-shits $i^{\text {, }}$ OAMB-Gal4/UAS-shits, and associated controls were isolated after eclosion and reared for $10-15 \mathrm{~d}$ at $18^{\circ} \mathrm{C}$ before test. Male flies were trained with a premated female at either $22^{\circ} \mathrm{C}$ (a permissive temperature) or $29^{\circ} \mathrm{C}$ (a restrictive temperature). After $1 \mathrm{~h}$ training, flies were immediately transferred to a new chamber containing a decapitated virgin and videotaped for $10 \mathrm{~min}$ at $22^{\circ} \mathrm{C}$. Tdc2-Gal4/ UAS-TrpA, OAMB-Gal4/UAS-TrpA, and associated controls were isolated after eclosion and reared for $6-10 \mathrm{~d}$ at $22^{\circ} \mathrm{C}$ before test. For courtship, a male fly was introduced into a courtship chamber at either $22^{\circ} \mathrm{C}$ or $29^{\circ} \mathrm{C}$ and paired with a virgin for scoring the CI. To test whether activation of OA neurons could provoke courtship memory formation, Male flies were trained with a virgin at either $22^{\circ} \mathrm{C}$ or $29^{\circ} \mathrm{C}$. After $1 \mathrm{~h}$ training, flies were immediately transferred to a new chamber containing a decapitated virgin and videotaped for $10 \mathrm{~min}$ at $22^{\circ} \mathrm{C}$.

\section{Results}

OA pathway is involved in courtship conditioning

To assess the role of OA in courtship conditioning, we examined the behavior of null mutants lacking tyramine $\beta$ hydroxylase 
$(\mathrm{T} \beta \mathrm{H})$, an enzyme converting tyramine to OA (Monastirioti et al., 1996). Courtship conditioning in $\mathrm{T} \beta \mathrm{H}$ male mutants was significantly impaired when compared with the wild-type (wt) ( $p=0.0051$; Fig. $1 A)$. The phenotype of $\mathrm{T} \beta \mathrm{H}$ mutants could be rescued by expressing $\mathrm{T} \beta \mathrm{H}$ transgene under the control of Tdc2-Gal4 (Cole et al., 2005), which drives expression in octopaminergic neurons, further confirming that $\mathrm{OA}$ is indeed important for courtship conditioning (Fig. 1A).

To test the roles of octopaminergic neurons in courtship learning, we used Tdc2Gal4 to drive the expression of shibire $e^{\text {ts }}$ $\left(s h i^{t s}\right)$, a temperature-sensitive mutant of dynamin, to block synaptic transmission of OA neurons. All transgenic flies were raised at $18^{\circ} \mathrm{C}$ so that the function of octopaminergic neurons was not affected during development. Flies were then shifted to the nonpermissive temperature, $29^{\circ} \mathrm{C}$, for testing. We found that reversible silencing of octopaminergic neurons during the training stage impaired courtship learning $(p=$ 0.031 ; Fig. $1 B$ ), indicating that octopaminergic neurons are functionally required for courtship conditioning.

\section{Activation of octopaminergic neurons reduces male-female courtship and mimics the suppression cue for courtship conditioning}

Male flies trained with a mated female subsequently develop courtship suppression even toward virgin females, suggesting that aversive pheromonal cues can generalize the suppression effects to appetitive female pheromones (Siegel and Hall, 1979; Ejima et al., 2007). The involvement of OA in courtship conditioning poses the interesting question of whether $\mathrm{OA}$ is involved in processing the aversive cues or appetitive cues. Our previous observation that $\mathrm{T} \beta \mathrm{H}$ male mutants are indistinguishable from wt in courting virgins indicates that the perception of female pheromones (presumably appetitive) by males is not affected by OA (Zhou et al., 2008), leading to the hypothesis that OA plays a role in sensing aversive pheromones of mated females.

To test this hypothesis, octopaminergic neurons were activated by the expression of TrpA1 which is a Drosophila ion channel sensitive to moderate heat (Hamada et al., 2008). We asked whether activation of octopaminergic neurons could affect malefemale courtship. Activation of octopaminergic neurons mimicked the effect of anti-aphrodisiac pheromones and significantly reduced male courtship toward females $(p<0.0001$; Fig. $1 C)$.

To further test our hypothesis, we asked whether activation of octopaminergic neurons could mimic the effects of aversive cues in conditioning the male response to female pheromones. We trained a male fly with a virgin female. Normally, no courtship conditioning would form because there are no aversive cues present to suppress courtship. Interestingly, when octopaminergic neurons were activated during training with virgins, courtship conditioning was artificially produced ( $p=0.04$ for Tdc2-Gal4/ UAS-TrpA vs UAS-TrpA/+, $p<0.0001$ for Tdc2-Gal4/UASANOVA).
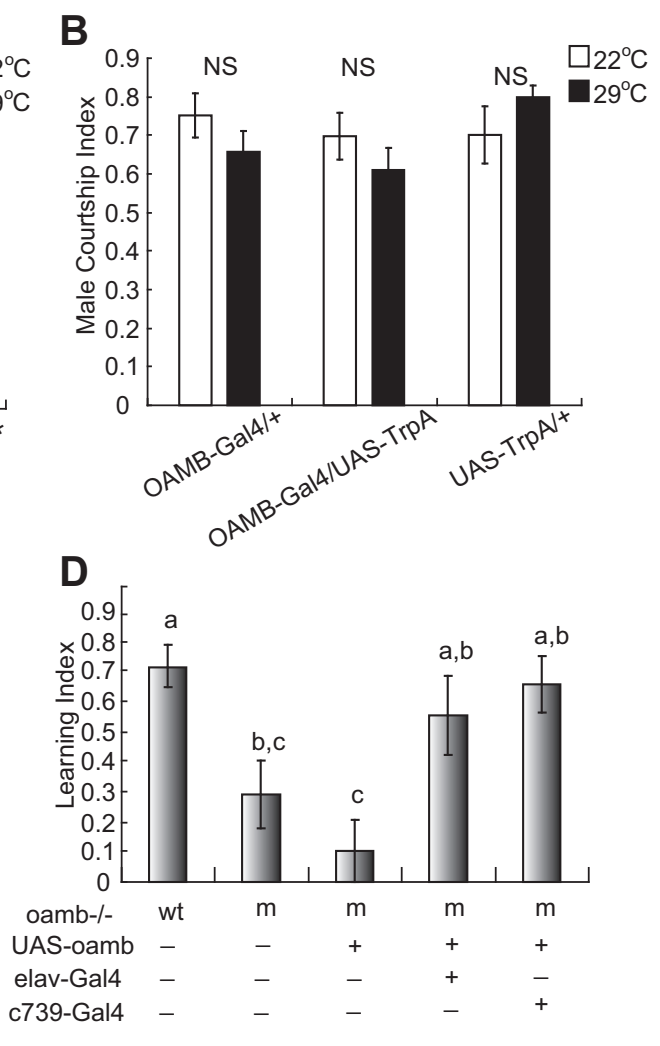

Figure 3. OAMB deletion or inactivation of OAMB neurons affects the formation of courtship memory. $A, \mathrm{Ll}$ of $\mathrm{OAMB}$-Gal4/UAS-shi and associated controls when trained at either $22^{\circ} \mathrm{C}$ or $29^{\circ} \mathrm{C} . N=11,12,23,19,11$, and 11 for each group; ${ }^{*} p<0.05$, NS $p>0.05$, Whitney test. B, Cl of OAMB-Gal4/UAS-TrpA and transgenic controls. No significant differences were detected (Mann-Whitne) virgin at $22^{\circ} \mathrm{C}$. No significant differences were detected (Mann-Whitney test): $N=21,22$, and 29 for each group. $D$, Rescue of OAMB mutant phenotype by expressing $0 A M B$ pan-neuronally or in mushroom body; wt denotes wild-type while $m$ denotes $0 a m b-1-$ background. $N=13,14,24,12$, and 12, respectively. Different letters above the bars indicate significant differences $(p<0.05$, one-way

TrpA vs Tdc2-Gal4/+; Fig. 1D). These results suggest that activation of octopaminergic neurons could mimic the aversive signals in courtship conditioning.

\section{The OAMB receptor and OAMB neurons are involved in courtship conditioning}

Four OA receptors are known: $1 \beta, 2 \beta, 3 \beta$, and OAMB (Balfanz et al., 2005; Maqueira et al., 2005). OAMB is expressed in the mushroom bodies and the central complex, suggesting a potential role in learning and memory (Han et al., 1998), although its only previously known function is in ovulation (Lee et al., 2003).

To label and manipulate the neurons in which OAMB is expressed, we created an OAMB-Gal4 line by targeting the insertion of the yeast transcription factor Gal4 to the OAMB locus (Fig. $2 A$ ). When OAMB-Gal4 was used to drive mCD8GFP expression, a subset of Kenyon cells was observed, with axonal bundles bifurcated only into $\alpha / \beta$ lobe (Fig. $2 B, C$ ), similar to the expression pattern of OAMB mRNA reported previously (Han et al., 1998), indicating the faithfulness of our OAMB-Gal4 line. Weak GFP expression was also detected in female oviducts and spermathecae (data not shown). No obvious sexual dimorphism of OAMB-Gal4 expression was detected (Fig. 2D,E).

To test the behavioral involvement of OAMB neurons in adults, $s h i^{t s}$ expression under the control of OAMB-Gal4 was used to acutely silence $\mathrm{OAMB}$ neurons. Inactivation of $\mathrm{OAMB}$ neurons reduced courtship conditioning, indicating that OAMB- 

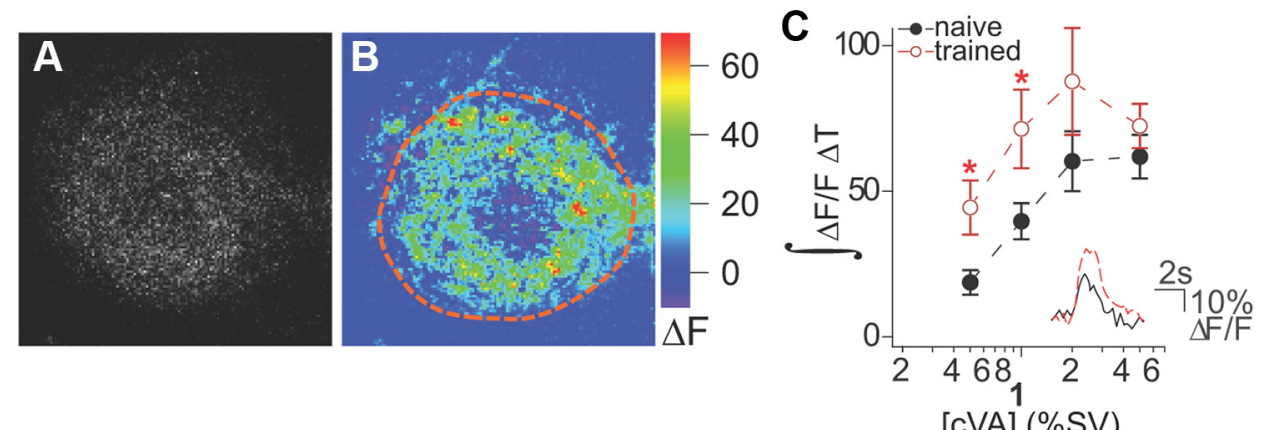

[cVA] (\%SV)
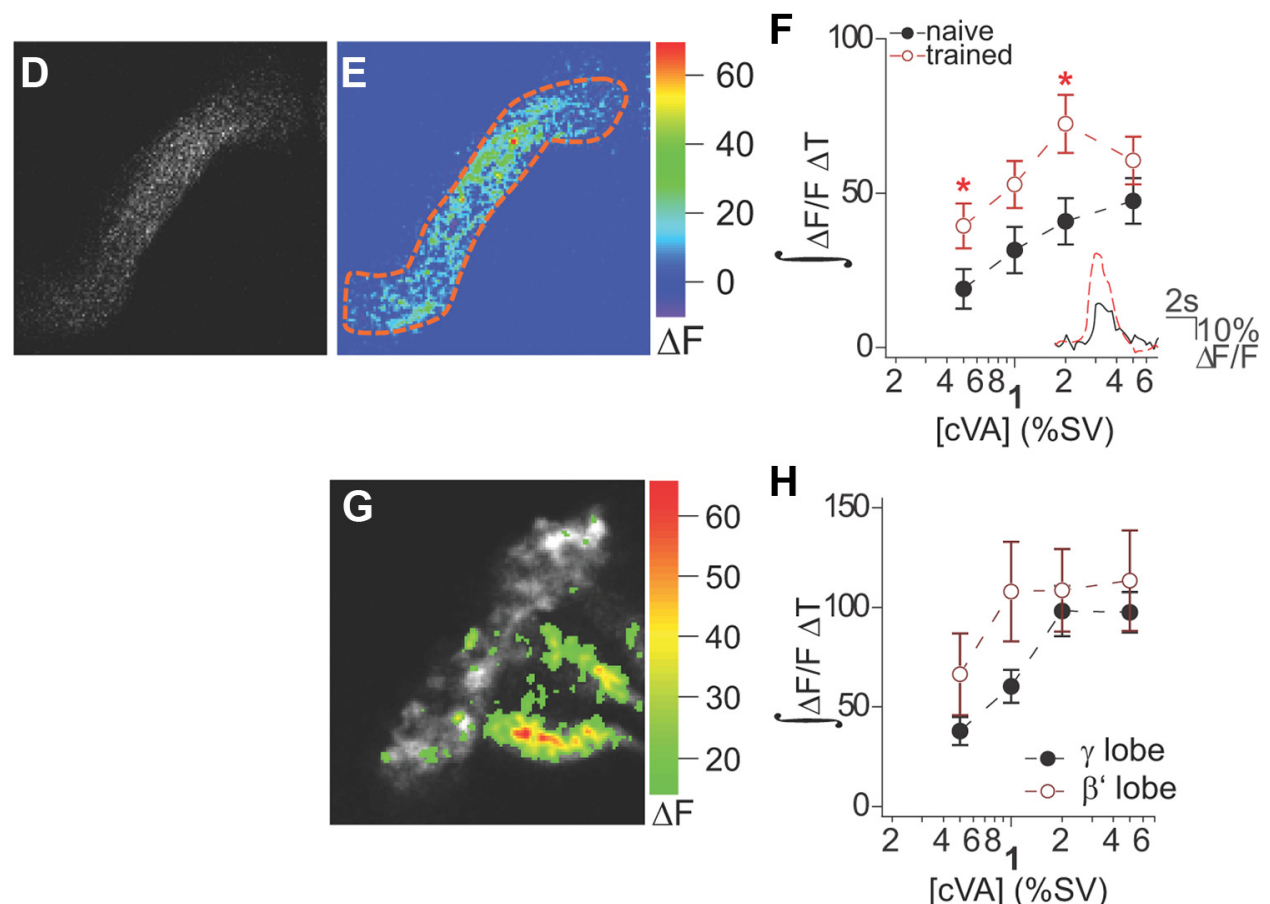

Figure 4. OAMB-Gal 4 neurons in the mushroom bodies respond to CVA stimuli. $A, B$, Dorsal view of $\alpha$ lobe from a male fly. Grayscale and pseudocolored images depict odor-evoked activity in OAMB neurons in response to delivery of CVA at 2\% saturated vapor pressure (SV). C, Responses to CVA in the $\alpha$ lobe of naive and trained male flies expressing G-CaMP3 and OAMB-Gal4. Graph displays mean integrated fluorescence change over time across a range of cVA concentrations for the $\alpha$ lobe. $n=9-15$ flies for each concentration. In the lower right corner of the graph, representative traces depict responses to $0.5 \%$ SV cVA as fluorescence change over time in the $\alpha$ lobes of both naive (black) and trained (red) male flies. D, E, Dorsal view of $\beta$ lobe from a naive male fly. Grayscale and pseudocolored images depict odor-evoked activity in OAMB neurons in response to delivery of cVA at 2\% SV. $\boldsymbol{F}$, Responses to cVA in the $\beta$ lobe of naive and trained male flies expressing G-CaMP3 and OAMB-Gal4. Graph displays mean integrated fluorescence change over time across a range of cVA concentrations for the $\beta$ lobe. $n=10-12$ flies for each concentration. In the lower right corner of the graph, representative traces depict responses to $0.5 \%$ SV cVA as fluorescence change over time in the $\beta$ lobes of both naive (black) and trained (red) male flies. $\mathbf{G}$, Optical section depicting Kenyon cell body layer and calyx labeled by G-CaMP3 and the OAMB-Gal4 line in a naive male fly. Grayscale image represents the average of all frames in the trial. Pseudocolored overlay represents odor-evoked responses to $2 \%$ SV cVA. $\boldsymbol{H}$, Responses to cVA in the $\beta^{\prime}$ and $\gamma$ lobes of naive male flies. Graph displays mean integrated fluorescence change over time across a range of cVA concentrations; $n=4-7$ flies for each concentration. Asterisk $\left(^{*}\right)$ denotes $p<0.05$. Error bars indicate SEM.

positive Kenyon cells were involved in courtship conditioning $(p=0.031$; Fig. $3 A)$.

Activation of OAMB neurons by TrpA1 expression under OAMB-Gal4 neither suppressed male courtship of females nor induced a courtship memory in the presence of decapitated virgins, suggesting that OAMB neurons only affect experiencedependent aspects of courtship conditioning (Fig. $3 B, C$ ).

To assess the involvement of OAMB in courtship conditioning, we performed behavioral tests on an oamb deletion mutant (Lee et al., 2003). Compared with the wt, the courtship learning index of oamb mutants was reduced (Fig. 3D). Furthermore, the courtship conditioning phenotype of oamb mutants could be rescued by expressing OAMB with either a pan-neuronal or a mushroom body-specific driver (Fig. 3D). These results indicate that $\mathrm{OAMB}$ is required for courtship conditioning.
OAMB neurons show dose-dependent responses to $\mathrm{CVA}$

cVA has been implicated as a key aversive pheromone in courtship conditioning (Ejima et al., 2007). To determine whether Kenyon cells labeled by the OAMB-Gal4 line responded to $\mathrm{cVA}$, we expressed the genetically encoded calcium indicator G-CaMP3, driven by OAMB-Gal4, and imaged the Kenyon cell layer of the MB (Fig. $4 G$ ). We found that $10 \pm 1 \%$ of all neurons observed exhibited responses to cVA ( $n=6$ naive male flies). We next hypothesized that neurons labeled by the OAMB-Gal4 line may respond to and integrate information about cVA and rejection. To test this hypothesis, we measured Kenyon cell responses to $\mathrm{CVA}$ in both naive and trained males. In naive males, OAMBGal4-labeled neurons in $\alpha$ and $\beta$ lobes of the mushroom bodies responded robustly to $\mathrm{CVA}$ in a dose-dependent manner (Fig. $4 A-F)$. cVA responses were enhanced in OAMB-expressing neu- 

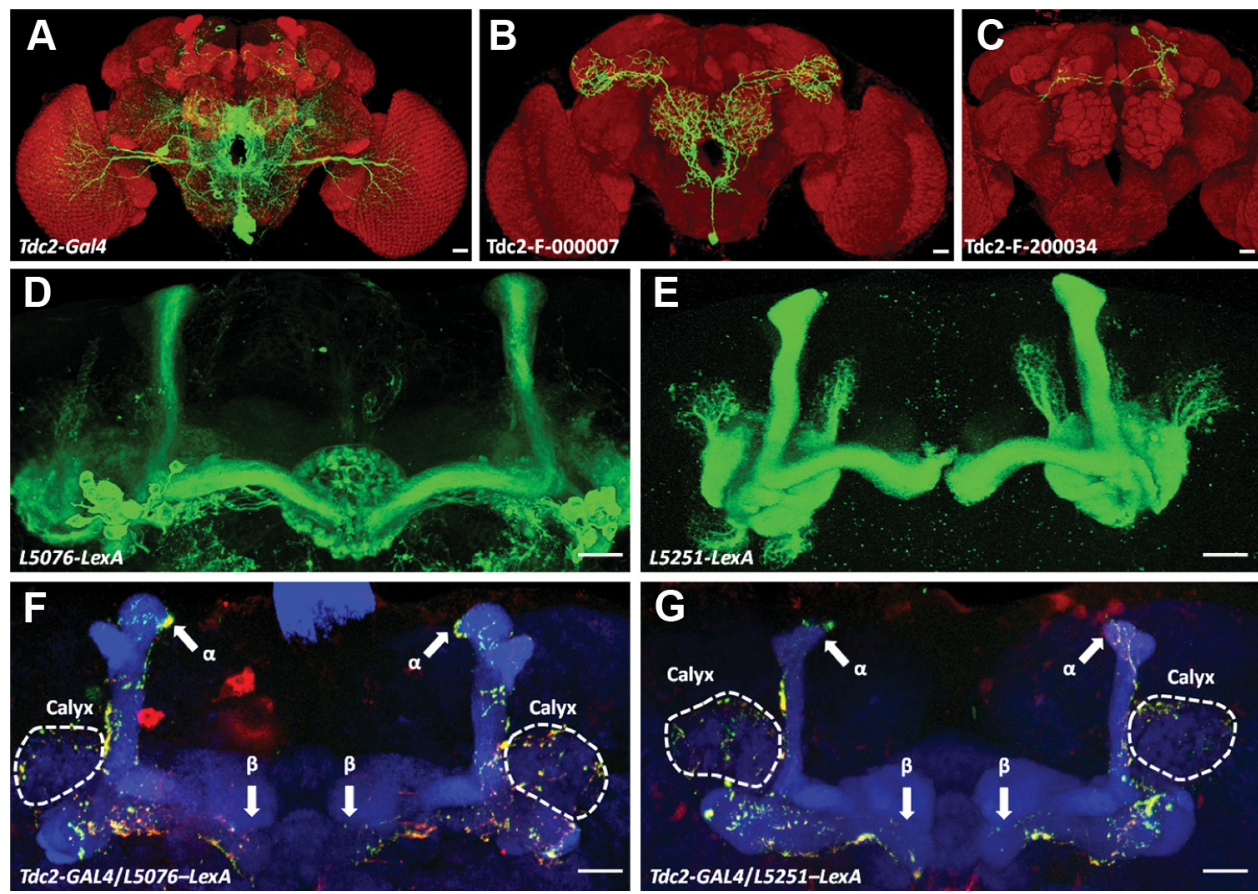

Figure 5. GRASP signals between Tdc2 neurons and $\alpha / \beta \mathrm{KCS}$. $A$, The expression pattern of Tdc2-Gal4. B, A single Tdc2 neuron innervating mushroom body calyx with VUM cell body. C, A single Tdc2 neuron innervating ipsilateral $\alpha$ and bilateral $\beta$ lobes in the mushroom bodies. Brain structures (red) are counterstained by anti-Dlg immunostaining and neurons are labeled by mCD8::GFP (A-C). Single neurons labeled by MARCM are obtained from FlyCircuit database (Chiang et al., 2011). D, E, Preferential expressions of two LexA lines in the mushroom bodies. Genotypes: L5076-LexA/+; LexAop-rCD2::GFP/+ (D); L5251-LexA/+; and LexAop-rCD2::GFP/+ (E).F, G, GRASP signals between TdC2 and LexA neurons. Tde2 neurons labeled by UAS-CD4::spGFP1-10 are visualized by anti-GFP immunostaining (red). LexA neurons labeled by LexAop-CD4:spGFP11 are invisible. Mushroom bodies are visualized by anti-Dlg immunostaining (blue). GRASP signals (green) occur sporadically in the calyx and $\alpha / \beta$ lobes, as expected. Scale bars: $20 \mu \mathrm{m}$. Genotypes: Tdc2-Gal4/L5076-LexA;UAS-CD4::spGFP1-10,LexAop-CD4:.spGFP11/+ (F) and Tdc2-Gal4/L5251-LexA;UAS-CD4::spGFP1-10,LexAop-CD4::spGFP11/+ (G) .

rons of trained male flies (Fig. 4C,F). These results suggest that cVA information is relayed to OAMB-expressing neurons in the mushroom bodies and responses in the OAMB neurons are enhanced by courtship memory training.

Clonal analysis of DA1 projections into the mushroom bodies suggests a preferential neuroanatomical connection with early $\alpha / \beta$ Kenyon cells in the calyx (Lin et al., 2007). Thus, we asked whether neurons labeled by the OAMB-Gal4 line were the only Kenyon cells to respond to the $\mathrm{cVA}$ odor stimulus. Using flies bearing the NP1131-Gal4 and UAS-GCaMP3 transgenes, we imaged $\gamma$ and $\beta^{\prime}$ lobe responses to increasing concentrations of cVA. Although $\beta^{\prime}$ lobe responses to cVA saturated earlier than the $\gamma$ lobe, all lobes examined exhibited dose-dependent responses to cVA (Fig. 4H). Thus, our functional analysis did not find that $\alpha / \beta$ Kenyon cells preferentially responded to DA1 inputs. In summary, although the OAMB neurons exhibit enhanced responses in trained males, this does not necessarily mean this subset of the $\alpha / \beta$ lobe is the only mushroom body region that is modified by courtship training.

\section{Octopaminergic neurons connect with neurons in the mushroom bodies}

OAMB receptor has been found to be highly enriched in the mushroom bodies, suggesting that mushroom bodies might be the direct synaptic targets of octopaminergic neurons. To test this hypothesis, we performed GFP reconstitution across synaptic partners (GRASP) analysis to examine whether octopaminergic neurons directly contacted mushroom body neurons (Feinberg et al., 2008). Tdc2-Gal4 was used to drive one-half of the split-GFP in octopaminergic neurons, while L5076 and L5251 were LexA strains driving the expression of the other half of split-GFP primarily in $\alpha / \beta$ lobes of the mushroom bodies. In both cases a significant fraction of GFP reconstitution signals was detected in the calyx region (Fig. $5 D-G$ ). Fur- ther studies by mosaic analysis with a repressible cell marker (MARCM) with Tdc2-Gal4 (Lee and Luo, 1999) showed that mushroom bodies were clearly innervated by single octopaminergic neurons (Fig. 5A-C). These results indicate that specific octopaminergic neurons form connections with mushroom bodies.

\section{Discussion}

We conclude that the neurotransmitter $\mathrm{OA}$ and its receptor OAMB expressed in neurons of the mushroom bodies are important for courtship conditioning.

In Drosophila, OA has been proposed to mediate the unconditioned reward stimuli in appetitive olfactory learning where sugar was used as the unconditioned stimulus while olfactory cues were used as conditioned stimuli (Schwaerzel et al., 2003). In honeybees, electric stimulation of VUMmx1 neurons could substitute food reward in proboscis extension reflex to modify olfactory responses (Hammer, 1993). It is unclear whether OA mediates general reward signals in insects as dopamine does in mammals, or it simply associates a specific type of unconditioned stimuli (sugar in case of Drosophila) with odorant cues. An inactive mutant with reduced activity of tyrosine decarboxylase, which catalyzes the conversion of tyrosine to tyramine, displays abnormal courtship learning (O'Dell, 1994). T $\beta \mathrm{H}$ mutants were unable to repress the initiation of courtship behavior in the presence of mated females (Certel et al., 2007). Either T $\beta \mathrm{H}$ mutation or activation of octopaminergic neurons enhanced male-male courtship when a male tester was presented with male and female targets in a competitive courtship assay, suggesting that a balanced OA signaling is important for sexual discrimination in flies (Certel et al., 2007, 2010).

Our results support a role for $\mathrm{OA}$ in courtship conditioning. In courtship conditioning, anti-aphrodisiac pheromones such as cVA 
are associated with appetitive pheromones to modify male response to mated females (Ejima et al., 2007). Here we provide evidence supporting the notion that OA mediates the sensing of aversive stimuli in courtship conditioning: (1) activation of octopaminergic neurons mimics anti-aphrodisiac pheromones in repressing male-female courtship and (2) activation of octopaminergic neurons in the presence of virgins could induce courtship memory, which subsequently reduces male courtship of virgin females.

Mushroom bodies are known to be important for learning and memory (de Belle and Heisenberg, 1994; McBride et al., 1999). We hypothesize that the OAMB receptor may act downstream of OA signaling to regulate courtship conditioning. This has been supported by our data that either loss-of-function mutation in the oamb gene or inactivation of OAMB neurons leads to defective courtship conditioning. The OAMB-Gal4 we generated labels a cluster of $\alpha / \beta$ lobe neurons. It has been reported that DA1 glomeruli in antenna lobe respond to cVA and only send axon branches to the calyx region of early $\alpha / \beta$ lobe neurons (Lin et al., 2007; Datta et al., 2008). The fact that OAMB neurons in $\alpha / \beta$ lobes of the mushroom bodies respond to cVA suggests $\mathrm{OAMB}$ neurons as a potential sensory integration site of courtship conditioning. Interestingly, OAMB neurons show enhanced response to $\mathrm{CVA}$ in trained males compared with naive males, suggesting that neuronal plasticity in OAMB circuits might mediate courtship conditioning. However, Activation of OAMB neurons does not reduce male courtship behavior, suggesting that the inhibitory effects of octopaminergic neurons may be mediated by other downstream neuronal circuits.

It is possible that different subsets of octopaminergic neurons act upon different downstream receptor neurons to regulate different behaviors. In Drosophila, anterior superior medial octopaminergic cells in the medial protocerebrum promote wakefulness (Crocker et al., 2010), whereas a few ventral unpaired median (VUM) neurons with neurites ramifying the subesophageal ganglion were suggested to regulate aggression (Certel et al., 2007; Zhou et al., 2008). We have characterized two classes of octopaminergic neurons innervating mushroom bodies, both of which are morphologically distinct from the VUM neurons involved in aggression (Fig. $5 B, C$ ). These suggest that innate social behaviors and experience-dependent behaviors are mediated by separate populations of octopaminergic cells. However, It remains to be established which classes of octopaminergic cells mediate reward olfactory learning or social learning in Drosophila.

\section{References}

Balfanz S, Strünker T, Frings S, Baumann A (2005) A family of octopamine [corrected] receptors that specifically induce cyclic AMP production or $\mathrm{Ca} 2+$ release in Drosophila melanogaster. J Neurochem 93:440-451.

Certel SJ, Savella MG, Schlegel DC, Kravitz EA (2007) Modulation of Drosophila male behavioral choice. Proc Natl Acad Sci U S A 104:4706-4711.

Certel SJ, Leung A, Lin CY, Perez P, Chiang AS, Kravitz EA (2010) Octopamine neuromodulatory effects on a social behavior decision-making network in Drosophila males. PLOS One 5:e13248.

Chiang AS, et al (2011) Three-dimensional reconstruction of brain-wide wiring networks in Drosophila at single-cell resolution. Curr Biol 21:1-11.

Cole SH, Carney GE, McClung CA, Willard SS, Taylor BJ, Hirsh J (2005) Two functional but noncomplementing Drosophila tyrosine decarboxylase genes: distinct roles for neural tyramine and octopamine in female fertility. J Biol Chem 280:14948-14955.

Crocker A, Shahidullah M, Levitan IB, Sehgal A (2010) Identification of a neural circuit that underlies the effects of octopamine on sleep:wake behavior. Neuron 65:670-681.

Datta SR, Vasconcelos ML, Ruta V, Luo S, Wong A, Demir E, Flores J, Balonze K, Dickson BJ, Axel R (2008) The Drosophila pheromone cVA activates a sexually dimorphic neural circuit. Nature 452:473-477. de Belle JS, Heisenberg M (1994) Associative odor learning in Drosophila abolished by chemical ablation of mushroom bodies. Science 263:692-695.

Ejima A, Smith BP, Lucas C, Levine JD, Griffith LC (2005) Sequential learning of pheromonal cues modulates memory consolidation in trainerspecific associative courtship conditioning. Curr Biol 15:194-206.

Ejima A, Smith BP, Lucas C, van der Goes van Naters W, Miller CJ, Carlson JR, Levine JD, Griffith LC (2007) Generalization of courtship learning in Drosophila is mediated by cis-vaccenyl acetate. Curr Biol 17:599-605.

Feinberg EH, Vanhoven MK, Bendesky A, Wang G, Fetter RD, Shen K, Bargmann CI (2008) GFP Reconstitution Across Synaptic Partners (GRASP) defines cell contacts and synapses in living nervous systems. Neuron 57:353-363.

Gailey DA, Jackson FR, Siegel RW (1984) Conditioning mutations in Drosophila melanogaster affect an experience-dependent behavioral modification in courting males. Genetics 106:613-623.

Griffith LC, Verselis LM, Aitken KM, Kyriacou CP, Danho W, Greenspan RJ (1993) Inhibition of calcium/calmodulin-dependent protein kinase in Drosophila disrupts behavioral plasticity. Neuron 10:501-509.

Hamada FN, Rosenzweig M, Kang K, Pulver SR, Ghezzi A, Jegla TJ, Garrity PA (2008) An internal thermal sensor controlling temperature preference in Drosophila. Nature 454:217-220.

Hammer M (1993) An identified neuron mediates the unconditioned stimulus in associative olfactory learning in honeybees. Nature 366:59-63.

Han KA, Millar NS, Davis RL (1998) A novel octopamine receptor with preferential expression in Drosophila mushroom bodies. J Neurosci 18:3650-3658.

Joiner MA, Griffith LC (1999) Mapping of the anatomical circuit of CaM kinase-dependent courtship conditioning in Drosophila. Learn Mem 6:177-192.

Joiner MlA, Griffith LC (1997) CaM kinase II and visual input modulate memory formation in the neuronal circuit controlling courtship conditioning. J Neurosci 17:9384-9391.

Lee HG, Seong CS, Kim YC, Davis RL, Han KA (2003) Octopamine receptor OAMB is required for ovulation in Drosophila melanogaster. Dev Biol 264:179-190.

Lee T, Luo L (1999) Mosaic analysis with a repressible cell marker for studies of gene function in neuronal morphogenesis. Neuron 22:451-461.

Lin HH, Lai JS, Chin AL, Chen YC, Chiang AS (2007) A map of olfactory representation in the Drosophila mushroom body. Cell 128:1205-1217.

Maqueira B, Chatwin H, Evans PD (2005) Identification and characterization of a novel family of Drosophila beta-adrenergic-like octopamine G-protein coupled receptors. J Neurochem 94:547-560.

McBride SM, Giuliani G, Choi C, Krause P, Correale D, Watson K, Baker G, Siwicki KK (1999) Mushroom body ablation impairs short-term memory and long-term memory of courtship conditioning in Drosophila melanogaster. Neuron 24:967-977.

Mehren JE, Ejima A, Griffith LC (2004) Unconventional sex: fresh approaches to courtship learning. Curr Opin Neurobiol 14:745-750.

Monastirioti M, Linn CE Jr, White K (1996) Characterization of Drosophila tyramine beta-hydroxylase gene and isolation of mutant flies lacking octopamine. J Neurosci 16:3900-3911.

O'Dell KM (1994) The inactive mutation leads to abnormal experiencedependent courtship modification in male Drosophila melanogaster. Behav Genet 24:381-388.

Rong YS, Golic KG (2000) Gene targeting by homologous recombination in Drosophila. Science 288:2013-2018.

Schwaerzel M, Monastirioti M, Scholz H, Friggi-Grelin F, Birman S, Heisenberg M (2003) Dopamine and octopamine differentiate between aversive and appetitive olfactory memories in Drosophila. J Neurosci 23:10495-10502.

Siegel RW, Hall JC (1979) Conditioned responses in courtship behavior of normal and mutant Drosophila. Proc Natl Acad Sci U S A 76:3430-3434.

Sitnik NA, Tokmacheva EV, Savvateeva-Popova EV (2003) The ability of Drosophila mutants with defects in the central complex and mushroom bodies to learn and form memories. Neurosci Behav Physiol 33:67-71.

Siwicki KK, Riccio P, Ladewski L, Marcillac F, Dartevelle L, Cross SA, Ferveur JF (2005) The role of cuticular pheromones in courtship conditioning of Drosophila males. Learn Mem 12:636-645.

Wang JW, Wong AM, Flores J, Vosshall LB, Axel R (2003) Two-photon calcium imaging reveals an odor-evoked map of activity in the fly brain. Cell 112:271-282.

Zhou C, Rao Y, Rao Y (2008) A subset of octopaminergic neurons are important for Drosophila aggression. Nat Neurosci 11:1059-1067. 